\title{
Bayes Estimation of Shape Parameter of Length Biased Weibull Distribution
}

\author{
Arun Kumar Rao' ${ }^{1}$ Himanshu Pandey ${ }^{2}$
}

1,2Department of Mathematics \& Statistics, DDU Gorakhpur University, Gorakhpur, INDIA himanshu pandey62@yahoo.com

\begin{tabular}{l}
\hline \hline \\
\hline Article History: \\
Received : 24-10-2020 \\
Revised : 18-01-2021 \\
Accepted : 19-01-2021 \\
Online : 15-04-2021 \\
\hline
\end{tabular}

\section{Keyword:}

Bayesian method; Length-biased Weibull distribution; Prior distributions; Loss functions.

\section{ABSTRACT}

In this paper, length biased Weibull distribution is considered for Bayesian analysis. The expressions for Bayes estimators of the parameter have been derived under squared error, precautionary, entropy, K-loss, and Al-Bayyati's loss functions by using quasi and gamma priors.

\section{A. INTRODUCTION}

The length biased Weibull distribution has been proposed by Pandya et al (Pandya, 2013). They obtained the Bayes estimators of the parameters under linex loss function. The probability density function of length biased Weibull distribution is given by

$$
f(x ; \theta)=\frac{\lambda^{2}}{\Gamma\left(\frac{1}{\lambda}\right)} \theta^{\left(1+\frac{1}{\lambda}\right)} x^{\lambda} e^{-\theta x^{\lambda}} \quad ; x>0 .
$$

The joint density function or likelihood function of (1) is given by

$$
f(\underline{x} ; \theta)=\lambda^{2 n}\left(\Gamma\left(\frac{1}{\lambda}\right)\right)^{-n} \theta^{n\left(1+\frac{1}{\lambda}\right)}\left(\prod_{i=1}^{n} x_{i}^{\lambda}\right) e^{-\theta \sum_{i=1}^{n} x_{i}^{\lambda}} .
$$

The log likelihood function is given by

$$
\log f(\underline{x} ; \theta)=2 n \log \lambda-n \log \left(\Gamma\left(\frac{1}{\lambda}\right)\right)+n\left(1+\frac{1}{\lambda}\right) \log \theta+\log \left(\prod_{i=1}^{n} x_{i}^{\lambda}\right)-\theta \sum_{i=1}^{n} x_{i}^{\lambda} .
$$


Differentiating (3) with respect to $\theta$ and equating to zero, we get the maximum likelihood estimator of $\theta$ which is given as

$$
\hat{\theta}=n\left(\sum_{i=1}^{n} x_{i}^{\lambda}\right)^{-1} \text {. }
$$

\section{B. METHODS}

The Bayesian inference procedures have been developed generally under squared error loss function

$$
L(\hat{\theta}, \theta)=(\hat{\theta}-\theta)^{2} .
$$

The Bayes estimator under the above loss function, say, $\hat{\theta}_{s}$ is the posterior mean, i.e,

$$
\hat{\theta}_{S}=E(\theta)
$$

Zellner (Zellner, 1986), Basu and Ebrahimi (Basu \& Ebrahimi, 1991) have recognized that the inappropriateness of using symmetric loss function. Norstrom (Norström, 1996) introduced precautionary loss function is given as

$$
L(\hat{\theta}, \theta)=\frac{(\hat{\theta}-\theta)^{2}}{\hat{\theta}} .
$$

The Bayes estimator under this loss function is denoted by $\hat{\theta}_{P}$ and is obtained as

$$
\hat{\theta}_{P}=\left[E\left(\theta^{2}\right)\right]^{1 / 2} \text {. }
$$

Calabria and Pulcini (Calabria \& Pulcini, 1996) points out that a useful asymmetric loss function is the entropy loss

$$
L(\delta) \propto\left[\delta^{p}-p \log _{e}(\delta)-1\right]
$$

where $\delta=\frac{\hat{\theta}}{\theta}$, and whose minimum occurs at $\hat{\theta}=\theta$. Also, the loss function $L(\delta)$ has been used in Dey et al. (Dey, Ghosh, \& Srinivasan, 1986) and Dey and Liu (Dey \& Pei-San Liao Liu, 1992), in the original form having $p=1$. Thus $L(\delta)$ can written be as

$$
L(\delta)=b\left[\delta-\log _{e}(\delta)-1\right] ; b>0 .
$$

The Bayes estimator under entropy loss function is denoted by $\hat{\theta}_{E}$ and is obtained by solving the following equation

$$
\hat{\theta}_{E}=\left[E\left(\frac{1}{\theta}\right)\right]^{-1} .
$$

Wasan (Melin, 1994) proposed the K-loss function which is given as

$$
L(\hat{\theta}, \theta)=\frac{(\hat{\theta}-\theta)^{2}}{\hat{\theta} \theta} .
$$

Under K-loss function the Bayes estimator of $\theta$ is denoted by $\hat{\theta}_{K}$ and is obtained as 


$$
\hat{\theta}_{K}=\left[\frac{E(\theta)}{E(1 / \theta)}\right]^{\frac{1}{2}} .
$$

Al-Bayyati (Al-Bayyati, 2002) introduced a new loss function which is given as

$$
L(\hat{\theta}, \theta)=\theta^{c}(\hat{\theta}-\theta)^{2}
$$

Under Al-Bayyati's loss function the Bayes estimator of $\theta$ is denoted by $\hat{\theta}_{A l}$ and is obtained as

$$
\hat{\theta}_{A l}=\frac{E\left(\theta^{c+1}\right)}{E\left(\theta^{c}\right)} .
$$

Several models have been investigated in last few years and a number of symmetric and asymmetric loss functions have been shown to be functional; we refer readers to (Nassir \& Ibrahim, 2020), (Ramos, Louzada, Ramos, \& Dey, 2019), (Reshi, Ahmad, \& Ahmad, 2019), (Dar, Ahmed, \& Reshi, 2018), (Ajami \& Jahanshahi, 2017), (Oluwafemi, 2017), (Mudasir Sofi, Ahamad, S.P., Ahamad, A., 2016), (Ahmad, Ahmad, \& Ahmed, 2016), (Tahir, 2015), (Reshi, J.A., Ahmad, A. and Mir, K.A., 2014), (Alzaatreh, Famoye, \& Lee, 2013).

Let us consider two prior distributions of $\theta$ to obtain the Bayes estimators.

(i) Quasi-prior: For the situation where we have no prior information about the parameter $\theta$, we may use the quasi density as given by

$$
g_{1}(\theta)=\frac{1}{\theta^{d}} ; \theta>0, d \geq 0,
$$

where $d=0$ leads to a diffuse prior and $d=1$, a non-informative prior.

(ii) Gamma prior: Generally, the gamma density is used as prior distribution of the parameter $\theta$ given by

$$
g_{2}(\theta)=\frac{\beta^{\alpha}}{\Gamma(\alpha)} \theta^{\alpha-1} e^{-\beta \theta} ; \theta>0 .
$$

\section{RESULT AND DISCUSSION}

\section{Posterior Density Under $g_{1}(\theta)$}

The posterior density of $\theta$ under $g_{1}(\theta)$, on using (2), is given by

$$
\begin{aligned}
f(\theta / \underline{x}) & =\frac{\lambda^{2 n}\left(\Gamma\left(\frac{1}{\lambda}\right)\right)^{-n} \theta^{n\left(1+\frac{1}{\lambda}\right)}\left(\prod_{i=1}^{n} x_{i}^{\lambda}\right) e^{-\theta \sum_{i=1}^{n} x_{i}^{\lambda}} \theta^{-d}}{\int_{0}^{\infty} \lambda^{2 n}\left(\Gamma\left(\frac{1}{\lambda}\right)\right)^{-n} \theta^{n\left(1+\frac{1}{\lambda}\right)}\left(\prod_{i=1}^{n} x_{i}^{\lambda}\right) e^{-\theta \sum_{i=1}^{n} x_{i}^{\lambda}} \theta^{-d} d \theta} \\
& =\frac{\theta^{n+\frac{n}{\lambda}-d} e^{-\theta \sum_{i=1}^{n} x_{i}^{\lambda}}}{\int_{0}^{\infty} \theta^{n+\frac{n}{\lambda}-d} e^{-\theta \sum_{i=1}^{n} x_{i}^{\lambda}} d \theta}
\end{aligned}
$$




$$
=\frac{\left(\sum_{i=1}^{n} x_{i}^{\lambda}\right)^{n+\frac{n}{\lambda}-d+1}}{\Gamma\left(n+\frac{n}{\lambda}-d+1\right)} \theta^{n+\frac{n}{\lambda}-d} e^{-\theta \sum_{i=1}^{n} x_{i}^{\lambda}}
$$

Theorem 1. On using (17), we have

$$
E\left(\theta^{c}\right)=\frac{\Gamma\left(n+\frac{n}{\lambda}-d+c+1\right)}{\Gamma\left(n+\frac{n}{\lambda}-d+1\right)}\left(\sum_{i=1}^{n} x_{i}^{\lambda}\right)^{-c} .
$$

Proof. By definition,

$$
\begin{aligned}
E\left(\theta^{c}\right)= & \int \theta^{c} f(\theta / \underline{x}) d \theta \\
& =\frac{\left(\sum_{i=1}^{n} x_{i}^{\lambda}\right)^{n+\frac{n}{\lambda}-d+1}}{\Gamma\left(n+\frac{n}{\lambda}-d+1\right)} \int_{0}^{\infty} \theta^{\left(n+\frac{n}{\lambda}-d+c\right)} e^{-\theta \sum_{i=1}^{n} x_{i}^{\lambda}} d \theta \\
& =\frac{\left(\sum_{i=1}^{n} x_{i}^{\lambda}\right)^{n+\frac{n}{\lambda}-d+1}}{\Gamma\left(n+\frac{n}{\lambda}-d+1\right)} \frac{\Gamma\left(n+\frac{n}{\lambda}-d+c+1\right)}{\left(\sum_{i=1}^{n} x_{i}^{\lambda}\right)^{n+\frac{n}{\lambda}-d+c+1}} \\
& =\frac{\Gamma\left(n+\frac{n}{\lambda}-d+c+1\right)}{\Gamma\left(n+\frac{n}{\lambda}-d+1\right)}\left(\sum_{i=1}^{n} x_{i}^{\lambda}\right)^{-c} .
\end{aligned}
$$

From equation (18), for $c=1$, we have

$$
E(\theta)=\left(n+\frac{n}{\lambda}-d+1\right)\left(\sum_{i=1}^{n} x_{i}^{\lambda}\right)^{-1} .
$$

From equation (18), for $c=2$, we have

$$
E\left(\theta^{2}\right)=\left[\left(n+\frac{n}{\lambda}-d+2\right)\left(n+\frac{n}{\lambda}-d+1\right)\right]\left[\sum_{i=1}^{n} x_{i}^{\lambda}\right]^{-2} .
$$

From equation (18), for $c=-1$, we have

$$
E\left(\frac{1}{\theta}\right)=\frac{1}{\left(n+\frac{n}{\lambda}-d\right)} \sum_{i=1}^{n} x_{i}^{\lambda} .
$$

From equation (18), for $c=c+1$, we have

$$
E\left(\theta^{c+1}\right)=\frac{\Gamma\left(n+\frac{n}{\lambda}-d+c+2\right)}{\Gamma\left(n+\frac{n}{\lambda}-d+1\right)}\left(\sum_{i=1}^{n} x_{i}^{\lambda}\right)^{-(c+1)} .
$$




\section{Bayes Estimators Under $g_{1}(\theta)$}

From equation (6), on using (19), the Bayes estimator of $\theta$ under squared error loss function is given by

$$
\hat{\theta}_{S}=\left(n+\frac{n}{\lambda}-d+1\right)\left(\sum_{i=1}^{n} x_{i}^{\lambda}\right)^{-1} .
$$

From equation (8), on using (20), the Bayes estimator of $\theta$ under precautionary loss function is obtained as

$$
\hat{\theta}_{P}=\left[\left(n+\frac{n}{\lambda}-d+2\right)\left(n+\frac{n}{\lambda}-d+1\right)\right]^{\frac{1}{2}}\left(\sum_{i=1}^{n} x_{i}^{\lambda}\right)^{-1} .
$$

From equation (10), on using (21), the Bayes estimator of $\theta$ under entropy loss function is given by

$$
\hat{\theta}_{E}=\left(n+\frac{n}{\lambda}-d\right)\left(\sum_{i=1}^{n} x_{i}^{\lambda}\right)^{-1}
$$

From equation (12), on using (19) and (21), the Bayes estimator of $\theta$ under K-loss function is given by

$$
\hat{\theta}_{K}=\left[\left(n+\frac{n}{\lambda}-d+1\right)\left(n+\frac{n}{\lambda}-d\right)\right]^{\frac{1}{2}}\left(\sum_{i=1}^{n} x_{i}^{\lambda}\right)^{-1} .
$$

From equation (14), on using (18) and (22), the Bayes estimator of $\theta$ under Al-Bayyati's loss function comes out to be

$$
\hat{\theta}_{A l}=\left(n+\frac{n}{\lambda}-d+c+1\right)\left(\sum_{i=1}^{n} x_{i}^{\lambda}\right)^{-1}
$$

\section{Posterior Density Under $g_{2}(\theta)$}

Under $g_{2}(\theta)$, the posterior density of $\theta$, using equation (2), is obtained as

$$
\begin{aligned}
f(\theta / \underline{x})= & \frac{\lambda^{2 n}\left(\Gamma\left(\frac{1}{\lambda}\right)\right)^{-n} \theta^{n\left(1+\frac{1}{\lambda}\right)}\left(\prod_{i=1}^{n} x_{i}^{\lambda}\right) e^{-\theta \sum_{i=1}^{n} x_{i}^{\lambda}} \frac{\beta^{\alpha}}{\Gamma(\alpha)} \theta^{\alpha-1} e^{-\beta \theta}}{\int_{0}^{\infty} \lambda^{2 n}\left(\Gamma\left(\frac{1}{\lambda}\right)\right)^{-n} \theta^{n\left(1+\frac{1}{\lambda}\right)}\left(\prod_{i=1}^{n} x_{i}^{\lambda}\right) e^{-\theta \sum_{i=1}^{n} x_{i}^{\lambda}} \frac{\beta^{\alpha}}{\Gamma(\alpha)} \theta^{\alpha-1} e^{-\beta \theta} d \theta} \\
= & \frac{\theta^{n+\frac{n}{\lambda}+\alpha-1} e^{-\left(\beta+\sum_{i=1}^{n} x_{i}^{\lambda}\right) \theta}}{\int_{0}^{\infty} \theta^{n+\frac{n}{\lambda}+\alpha-1} e^{-\left(\beta+\sum_{i=1}^{n} x_{i}^{\lambda}\right) \theta} d \theta} \\
= & \frac{\theta^{n+\frac{n}{\lambda}+\alpha-1} e^{-\left(\beta+\sum_{i=1}^{n} x_{i}^{\lambda}\right) \theta}}{\Gamma\left(n+\frac{n}{\lambda}+\alpha\right) /\left(\beta+\sum_{i=1}^{n} x_{i}^{\lambda}\right)^{n+\frac{n}{\lambda}+\alpha}}
\end{aligned}
$$




$$
=\frac{\left(\beta+\sum_{i=1}^{n} x_{i}^{\lambda}\right)^{n+\frac{n}{\lambda}+\alpha}}{\Gamma\left(n+\frac{n}{\lambda}+\alpha\right)} \theta^{n+\frac{n}{\lambda}+\alpha-1} e^{-\left(\beta+\sum_{i=1}^{n} x_{i}^{\lambda}\right) \theta}
$$

Theorem 2. On using (28), we have

$$
E\left(\theta^{c}\right)=\frac{\Gamma\left(n+\frac{n}{\lambda}+\alpha+c\right)}{\Gamma\left(n+\frac{n}{\lambda}+\alpha\right)}\left(\beta+\sum_{i=1}^{n} x_{i}^{\lambda}\right)^{-c} .
$$

Proof. By definition,

$$
\begin{aligned}
E\left(\theta^{c}\right) & =\int \theta^{c} f(\theta / \underline{x}) d \theta \\
& =\frac{\left(\beta+\sum_{i=1}^{n} x_{i}^{\lambda}\right)^{n+\frac{n}{\lambda}+\alpha}}{\Gamma\left(n+\frac{n}{\lambda}+\alpha\right)} \int_{0}^{\infty} \theta^{n+\frac{n}{\lambda}+\alpha+c-1} e^{-\left(\beta+\sum_{i=1}^{n} x_{i}^{\lambda}\right) \theta} d \theta \\
& =\frac{\left(\beta+\sum_{i=1}^{n} x_{i}^{\lambda}\right)^{n+\frac{n}{\lambda}+\alpha}}{\Gamma\left(n+\frac{n}{\lambda}+\alpha\right)} \frac{\Gamma\left(n+\frac{n}{\lambda}+\alpha+c\right)}{\left(\beta+\sum_{i=1}^{n} x_{i}^{\lambda}\right)^{n+\frac{n}{\lambda}+\alpha+c}} \\
& =\frac{\Gamma\left(n+\frac{n}{\lambda}+\alpha+c\right)}{\Gamma\left(n+\frac{n}{\lambda}+\alpha\right)}\left(\beta+\sum_{i=1}^{n} x_{i}^{\lambda}\right)^{-c} .
\end{aligned}
$$

From equation (29), for $c=1$, we have

$$
E(\theta)=\left(n+\frac{n}{\lambda}+\alpha\right)\left(\beta+\sum_{i=1}^{n} x_{i}^{\lambda}\right)^{-1} .
$$

From equation (29), for $c=2$, we have

$$
E\left(\theta^{2}\right)=\left[\left(n+\frac{n}{\lambda}+\alpha+1\right)\left(n+\frac{n}{\lambda}+\alpha\right)\right]\left(\beta+\sum_{i=1}^{n} x_{i}^{\lambda}\right)^{-2} .
$$

From equation (29), for $c=-1$, we have

$$
E\left(\frac{1}{\theta}\right)=\frac{1}{\left(n+\frac{n}{\lambda}+\alpha-1\right)}\left(\beta+\sum_{i=1}^{n} x_{i}^{\lambda}\right) .
$$

From equation (29), for $c=c+1$, we have

$$
E\left(\theta^{c+1}\right)=\frac{\Gamma\left(n+\frac{n}{\lambda}+\alpha+c+1\right)}{\Gamma\left(n+\frac{n}{\lambda}+\alpha\right)}\left(\beta+\sum_{i=1}^{n} x_{i}^{\lambda}\right)^{-(c+1)} .
$$




\section{Bayes Estimators Under $g_{2}(\theta)$}

From equation (6), on using (30), the Bayes estimator of $\theta$ under squared error loss function is given by

$$
\hat{\theta}_{S}=\left(n+\frac{n}{\lambda}+\alpha\right)\left(\beta+\sum_{i=1}^{n} x_{i}^{\lambda}\right)^{-1}
$$

From equation (8), on using (31), the Bayes estimator of $\theta$ under precautionary loss function is obtained as

$$
\hat{\theta}_{P}=\left[\left(n+\frac{n}{\lambda}+\alpha+1\right)\left(n+\frac{n}{\lambda}+\alpha\right)\right]^{\frac{1}{2}}\left(\beta+\sum_{i=1}^{n} x_{i}^{\lambda}\right)^{-1} .
$$

From equation (10), on using (32), the Bayes estimator of $\theta$ under entropy loss function is given by

$$
\hat{\theta}_{E}=\left(n+\frac{n}{\lambda}+\alpha+1\right)\left(\beta+\sum_{i=1}^{n} x_{i}^{\lambda}\right)^{-1} .
$$

From equation (12), on using (30) and (32), the Bayes estimator of $\theta$ under K-loss function is given by

$$
\hat{\theta}_{K}=\left[\left(n+\frac{n}{\lambda}+\alpha\right)\left(n+\frac{n}{\lambda}+\alpha-1\right)\right]^{\frac{1}{2}}\left(\beta+\sum_{i=1}^{n} x_{i}^{\lambda}\right)^{-1} .
$$

From equation (14), on using (29) and (33), the Bayes estimator of $\theta$ under Al-Bayyati's loss function comes out to be

$$
\hat{\theta}_{A l}=\left(n+\frac{n}{\lambda}+\alpha+c\right)\left(\beta+\sum_{i=1}^{n} x_{i}^{\lambda}\right)^{-1} .
$$

\section{CONCLUSION AND SUGGESTIONS}

In this paper, we have obtained a number of estimators of parameter of length biased Weibull distribution. In equation (4) we have obtained the maximum likelihood estimator of the parameter. In equation (23), (24), (25), (26) and (27) we have obtained the Bayes estimators under different loss functions using quasi prior. In equation (34), (35), (36), (37) and (38) we have obtained the Bayes estimators under different loss functions using gamma prior. In the above equation, it is clear that the Bayes estimators depend upon the parameters of the prior distribution. We therefore recommend that the estimator's choice lies according

to the value of the prior distribution which in turn depends on the situation at hand.

\section{REFERENCES}

Ahmad, K., Ahmad, S. P., \& Ahmed, A. (2016). Classical and Bayesian approach in estimation of scale parameter of Nakagami distribution. Journal of Probability and Statistics, 2016. https://doi.org/10.1155/2016/7581918

Ajami, M., \& Jahanshahi, S. M. A. (2017). Parameter estimation in weighted Rayleigh distribution. Journal of Modern Applied Statistical Methods, 16(2), 256-276. https://doi.org/10.22237/jmasm/1509495240

Al-Bayyati. (2002). Comparing methods of estimating Weibull failure models using simulation. Baghdad University, Iraq.

Alzaatreh, A., Famoye, F., \& Lee, C. (2013). Weibull-pareto distribution and its applications. Communications in Statistics - Theory and Methods, 42(9), 1673-1691. 
https://doi.org/10.1080/03610926.2011.599002

Basu, A. P., \& Ebrahimi, N. (1991). Bayesian approach to life testing and reliability estimation using asymmetric loss function. Journal of Statistical Planning and Inference, 29(1-2), 2131. https://doi.org/10.1016/0378-3758(92)90118-C

Calabria, R., \& Pulcini, G. (1996). Point estimation under asymmetric loss functions for lefttruncated exponential samples. Communications in Statistics - Theory and Methods, 25(3), 585-600. https://doi.org/10.1080/03610929608831715

Dar, A. A., Ahmed, A., \& Reshi, J. A. (2018). Characterization and estimation of weighted Maxwell-Boltzmann distribution. Applied Mathematics and Information Sciences, 12(1), 193-202. https://doi.org/10.18576/amis/120119

Dey, D. K., Ghosh, M., \& Srinivasan, C. (1986). Simultaneous estimation of parameters under entropy loss. Journal of Statistical Planning and Inference, 15(C), 347-363. https://doi.org/10.1016/0378-3758(86)90108-4

Dey, D. K., \& Pei-San Liao Liu. (1992). On comparison of estimators in a generalized life model. Microelectronics Reliability, 32(1-2), 207-221. https://doi.org/10.1016/00262714(92)90099-7

Melin, J. B. (1994). Parametric estimation. Cost Engineering (Morgantown, West Virginia), 36(1), 19-24. https://doi.org/10.2307/2284262

Mudasir Sofi, Ahamad, S.P., Ahamad, A. (2016). Bayesian Estimation of Length Biased Nakagami Distribution. International Journal of Modern Mathematical Sciences, 44(2), 147-159.

Nassir, L. M., \& Ibrahim, N. A. (2020). The Bayesian estimator for probabilistic dagum distribution. International Journal of Innovation, Creativity and Change, 12(5), 42-74.

Norström, J. G. (1996). The use of precautionary loss functions in risk analysis. IEEE Transactions on Reliability, 45(3), 400-403. https://doi.org/10.1109/24.536992

Oluwafemi, O. S. (2017). Length and Area Biased Exponentiated Weibull Distribution Based on Forest Inventories. Biometrics \& Biostatistics International Journal, 6(2). https://doi.org/10.15406/bbij.2017.06.00163

Pandya, M., Pandya, S. and Andharia, P. (2013). Bayes estimation of Weibull length biased distribution. Asian Journal of Current Engineering and Maths, 2(1), 44-49.

Ramos, P. L., Louzada, F., Ramos, E., \& Dey, S. (2019). The Fréchet distribution: Estimation and application - An overview. Journal of Statistics and Management Systems, 1-30. https://doi.org/10.1080/09720510.2019.1645400

Reshi, J.A., Ahmad, A. and Mir, K.A. (2014). Characterizations and estimation in the lengthbiased generalized Rayleigh distribution. Mathematical Theory and Modeling, 4(6), 87-98.

Reshi, J. A., Ahmad, A., \& Ahmad, S. P. (2019). Bayesian Inference of Ailamujia Distribution using Different Loss Functions. In Bayesian Analysis and Reliability Estimation of Generalized Probability Distributions (pp. 41-49). https://doi.org/10.21467/books.44.4

Tahir, M.H, Cordeiro, G.M. and Zubair, M. (2015). The Weibull-Lomax distribution: properties and applications. Hacettepe Journal of Mathematics and Statistics, 44(2), 461-480.

Zellner, A. (1986). Bayesian Estimation and Prediction Using Asymmetric Loss Functions. Journal of the American Statistical Association, 81(394), 446. https://doi.org/10.2307/2289234 